\title{
The effect of epidural analgesia on maternal-neonatal outcomes: a retrospective study
}

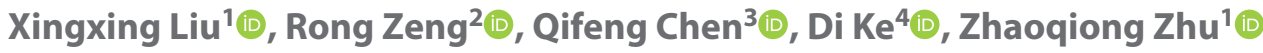 \\ ${ }^{1}$ Department of Anesthesiology, Affiliated Hospital of Zunyi Medical University, Zunyi, Guizho, China \\ ${ }^{2}$ School of Public Health, Zunyi Medical University, Zunyi , Guizhou, China \\ ${ }^{3}$ Department of Obstetrics, Affiliated Hospital of Zunyi Medical University, Zunyi, Guizhou, China \\ ${ }^{4}$ Department of Intervention, Affiliated Hospital of Zunyi Medical University, Zunyi, Guizhou, China
}

\begin{abstract}
Objectives: Epidural analgesia is commonly used for relieving labor pain in contemporary clinical practice. The rate of pregnant women who request epidural analgesia during labor has been increasing annually, leading to a debate on the effect of epidural analgesia on maternal or neonatal outcomes.

Material and methods: The medical records of nulliparous women with a term singleton pregnancy from January to December 2019 at the Affiliated Hospital of Zunyi Medical University were retrospectively reviewed. The women were divided into those who received epidural analgesia during delivery and those who did not receive it. Maternal and neonatal outcomes were assessed.

Results: A total of 528 women met the inclusion criteria. The overall labor analgesia rate was $43.0 \%$ (227). Women with epidural analgesia had a significantly longer second stage [34.5 (22.8-65.3) vs $27.0(18.0-41.3) \mathrm{min}, \mathrm{p}<0.001]$ and total duration of labor [698.5 (493.5-875.0) vs 489.5 (344.0-676.3) $\mathrm{min}, \mathrm{p}<0.001]$ compared with those without epidural. There were no significant relationships between epidural analgesia and the normal vaginal delivery rate, the incidence of episiotomy, and other adverse maternal or neonatal outcomes ( $p>0.05)$.

Conclusions: Epidural analgesia can prolong the second stage of labor, but this is no increased risk for both mother and neonate.
\end{abstract}

Key words: labor epidural analgesia; maternal and neonatal outcome

Ginekologia Polska 2021; 92, 9: 637-641

\section{INTRODUCTION}

Labor pain is one of the most common pains, which could cause a series of neurophysiological changes such as the increase of maternal oxygen stress hormones, the increase of blood pressure and the decrease of fetal oxygen transport, thus may affecting maternal and fetal well-being [1].

Epidural analgesia (EA) is currently one of the most effective ways of providing excellent intrapartum analgesia for pregnant women $[2,3]$. In recent years, the rate of parturients who request EA during labor has widely increased, with a rate of $20-70 \%$ of all deliveries [4-7]. Since introduction of EA into the field of labor analgesia, attention has been paid to the effect of EA and pain relief in labor on maternal and neonatal outcomes. However, this effect of EA and pain relief in labor is controversial. Several studies have shown that beneficial analgesic effects come at the expense of pro- longed labor, and an increase in instrumental vaginal delivery and emergency cesarean section, among others [8-11]. With regard to analgesic effects on the newborn, the existing literature on the relationship between EA and neonatal outcomes are equivocal [12-15]. In addition, whether the increase in maternal or neonatal morbidity is a consequence of EA is unclear. Anesthetic effects on maternal and neonatal outcomes continue to be investigated. Current and future work in these areas may improve clinicians' ability to individualize obstetric anesthesia treatment.

The aim of this study was to determine the effect of EA on maternal-neonatal outcomes regarding the period of labor.

\section{MATERIAL AND METHODS}

The study was conducted in accordance with the Declaration of Helsinki. The study protocol was approved by

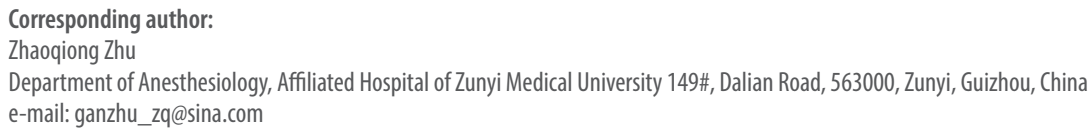


the Ethics Committee of the Affiliated Hospital of Zunyi Medical University, Guizhou, China (KLL-2020-015). Informed consent was waived owing to the retrospective nature of the study design. The study was performed in the Affiliated Hospital of Zunyi Medical University.

The reference population was composed of a group of nulliparous women with a term singleton pregnancy ( $\geq 37$ weeks' gestation) who received care during childbirth in the Affiliated Hospital of Zunyi Medical University from January to December 2019. The exclusion criteria were as follows: planned surgical delivery (elective cesarean section), multipara, gestation < 37 weeks, multiple pregnancies, intrauterine fetal demise, and other anesthetic combinations. The study population was determined using these criteria of exclusion (Fig. 1).

Mothers were divided into the epidural and non-epidural groups according to whether they received analgesia by their choice. EA was performed in women when they requested it, regardless of the size of cervical dilatation during the labor period. The epidural space was located between $\mathrm{L} 2$ and $\mathrm{L} 3$, and the epidural catheter was inserted into the epidural space for $3 \mathrm{~cm} .8 \mathrm{~mL} 0.1 \%$ ropivacaine with $4 \mu \mathrm{g}$ sufentanil was given as loading dose. An epidural catheter was situated and connected to a patient-controlled epidural analgesia (PCEA) pump containing solutions of ropivacaine $0.1 \%$ with sufentanil $0.5 \mu \mathrm{g} / \mathrm{mL}$. The PCEA regimen was as follows: $8 \mathrm{~mL} / \mathrm{h}$ for continuous infusion, $8 \mathrm{~mL}$ at a bolus dose, and $30 \mathrm{~min}$ for locking [16]. PCEA was stopped at the end of the second stage of labor, and the catheter was removed two hours after delivery.

To collect the data, we used electronic medical records of the patients under study. Demographic data that were collected included maternal age, height, weight, gesta- tional weeks, and complications. Maternal complications were defined as follows. Hypertension included essential or gestational hypertension or pre-eclampsia. Diabetes was defined as gestational diabetes, type 1 diabetes or type 2 diabetes. Abnormal results of thyroid function examination could be diagnosed as thyroid disease. Anemia was defined as hemoglobin concentration $<110 \mathrm{~g} / \mathrm{L}$. Maternal outcomes included the type of delivery (spontaneous or instrumental vaginal delivery, or emergency cesarean section), meconium-stained amniotic fluid, episiotomy, duration of the first and second stages of labor and the total duration of labor, the amount of postpartum hemorrhage, and length of postpartum hospital stay. Neonatal data included the Apgar score at 1, 5, and $10 \mathrm{~min}$, a 5-min Apgar score $\leq 7$, and neonatal intensive care unit admission.

Statistical analyses were performed using the Statistical Package for Social Sciences (SPSS ${ }^{\mathrm{TM}}$ ), Windows version 23.0 (IBM Corp., Armonk, NY, USA). First, the Shapiro-Wilk test was used to assess normal distribution of the data. Because of a lack of agreement with normal distribution, descriptive statistics analysis was performed using frequencies and percentages for categorical variables and the median and interquartile range for quantitative variables. Bivariate analysis was then performed on the obstetric history and maternal or neonatal outcomes with use of EA (no/yes), using Fisher's exact test or Pearson's chi-square test for categorical variables and the Mann-Whitney $U$ test for quantitative variables. For statistical tests, a value of $\mathrm{p}<0.05$ was considered to indicate statistical significance.

\section{RESULTS}

The reference population consisted of 4085 pregnant women of whom 2296 (56.2\%) were excluded for elective

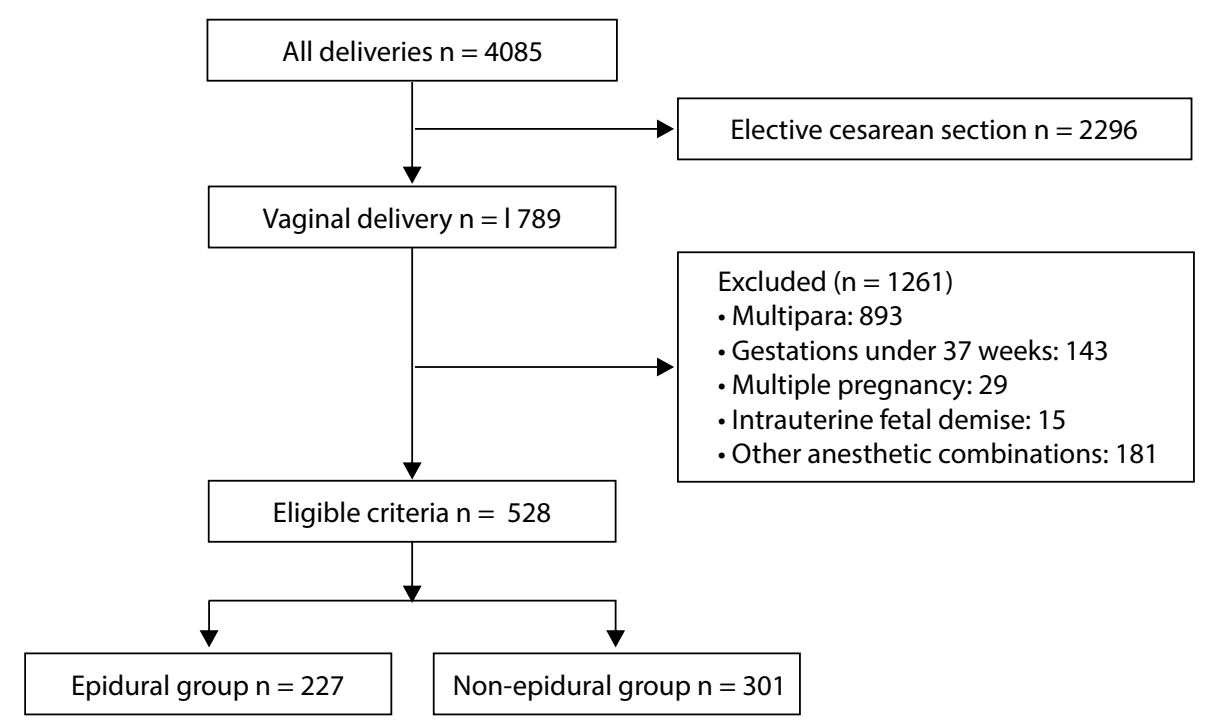

Figure 1. Flow chart of women in the study 
cesarean section and 1261 (30.9\%) for various reasons, with a final total of $528(12.9 \%)$ pregnant women. The study cohort was divided into the epidural group (227 women) and non-epidural group (301 women). The overall epidural rate was $43.0 \%$ (Fig. 1).

Women who received EA were more likely to be older compared with those without an epidural $(p<0.01)$. There were no differences in maternal height, weight, gestational age, and complications between the two groups. Demographics of the women are shown in Table 1.

Maternal outcomes are shown in Table 2. The times of the first $(p<0.001)$ and second $(p<0.001)$ stages of labor were longer in the epidural group than in the non-epidural group. Additionally, the total duration of labor was significantly longer in the epidural group than in the non-epidural group ( $p<0.001$ ). However, there was no significant difference in the method of delivery between the groups. Additionally, the rate of spontaneous vaginal delivery. The amount of postpartum hemorrhage and the mother's hospital stay after delivery were not significantly different between the groups.

Regarding neonatal outcomes, there were no significant differences in the Apgar score at 1, 5, and $10 \mathrm{~min}$, and admission to the neonatal ward between the two groups (Tab. 3).

\section{DISCUSSION}

In this study, we found that EA was associated with longer duration of the second and total stages of labor. However, this did not affect the normal vaginal delivery rate, the inci-

\begin{tabular}{|c|c|c|c|}
\hline \multirow{2}{*}{ Variables } & \multicolumn{2}{|c|}{ Epidural Analgesia } & \multirow{2}{*}{ p value } \\
\hline & No $(n=301)$ & Yes $(n=227)$ & \\
\hline Maternal age [years] & $26.0(23.0-28.0)$ & $27.0(25.0-29.0)$ & $<0.01$ \\
\hline Maternal height $[\mathrm{cm}]$ & $158.0(155.5-160.0)$ & $158.0(155.0-162.0)$ & 0.29 \\
\hline Maternal weight [kg] & $67.0(60.0-72.3)$ & $67.0(62.0-72.0)$ & 0.66 \\
\hline Gestational week, [weeks \pm days] & $39.6(38.9-40.3)$ & $39.7(39.0-40.3)$ & 0.19 \\
\hline \multicolumn{4}{|l|}{ Complications } \\
\hline Hypertension & $6(2.0)$ & $3(1.3)$ & 0.56 \\
\hline Diabetes & $32(10.6)$ & $33(14.5)$ & 0.18 \\
\hline Thyroid disorders & $37(12.3)$ & $25(11.0)$ & 0.65 \\
\hline Anemia & $56(18.6)$ & $28(12.3)$ & 0.05 \\
\hline
\end{tabular}

Data are presented as $\mathrm{n}(\%)$ or median $\left(25^{\text {th }}-75^{\text {th }}\right.$ percentiles); Diabetes - gestational diabetes mellitus, type 1 diabetes mellitus, or type 2 diabetes mellitus; hypertension - essential or gestational hypertension or pre-eclampsia; thyroid disorders - abnormal results of thyroid function tests; anemia — a concentration of hemoglobin $<110 \mathrm{~g} / \mathrm{L}$

\begin{tabular}{|c|c|c|c|}
\hline \multirow{2}{*}{ Variables } & \multicolumn{2}{|c|}{ Epidural Analgesia } & \multirow{2}{*}{ p value } \\
\hline & No $(n=301)$ & Yes $(n=227)$ & \\
\hline \multicolumn{4}{|l|}{ Type of delivery, n (\%) } \\
\hline Normal Vaginal & $241(80.0)$ & $196(86.3)$ & 0.06 \\
\hline Instrumental & $5(1.7)$ & $2(0.9)$ & 0.44 \\
\hline Emergency CS & $55(18.3)$ & $29(12.8)$ & 0.09 \\
\hline Meconium-stained amniotic fluid & $81(26.9)$ & $54(23.8)$ & 0.42 \\
\hline Episiotomy* & $122(49.6)$ & $92(46.5)$ & 0.51 \\
\hline First stage of labor duration [minutes] & $445.0(306.0-621.3)$ & $655.0(454.3-820.5)$ & $<0.001$ \\
\hline Second stage of labor duration [minutes] & $27.0(18.0-41.3)$ & $34.5(22.8-65.3)$ & $<0.001$ \\
\hline Total time of labor [minutes] & $489.5(344.0-676.3)$ & $698.5(493.5-875.0)$ & $<0.001$ \\
\hline \multicolumn{4}{|l|}{ Postpartum haemorrhage } \\
\hline$<0.5 \mathrm{~L}$ & $244(81.1)$ & $197(86.8)$ & 0.79 \\
\hline$\geq 0.5 \mathrm{~L}$ & $57(18.9)$ & $30(13.2)$ & 0.79 \\
\hline Maternal postnatal length of stay & $2(2-3)$ & $2(1-2)$ & 0.21 \\
\hline
\end{tabular}

CS - cesarean section; *Women who achieved a vaginal birth 


\begin{tabular}{|l|c|c|c|}
\hline Table 3. Neonatal outcomes \\
\hline \multirow{2}{*}{ Variables } & \multicolumn{2}{|c|}{ Epidural Analgesia } & p value \\
\hline Apgar score at $1 \mathrm{~min}$ & No $(\mathbf{n = 3 0 1 )}$ & Yes $(\mathbf{n = 2 2 7})$ & 0.56 \\
\hline Apgar score at $5 \mathrm{~min}$ & $10(10-10)$ & $10(10-10)$ & 0.93 \\
\hline Apgar score at $10 \mathrm{~min}$ & $10(10-10)$ & $10(10-10)$ & 0.88 \\
\hline 5-min Apgar score $\leq 7$ & $10(10-10)$ & $10(10-10)$ & 0.84 \\
\hline NICU admission & $1(0.3)$ & $1(0.4)$ & 0.98 \\
\hline
\end{tabular}

$\mathrm{NICU}$ - neonatal intensive care unit

dence of episiotomy, and other adverse maternal or neonatal outcomes. Our results are consistent with recent studies that EA during delivery does not increase the rate of vaginal delivery, and EA during delivery is safe for both mothers and fetuses $[17,18]$. A 2018 Cochrane review suggested that EA had no effect on the risk of cesarean section [19]. This review also showed that EA did not appear to have an immediate effect on neonatal status as determined by Apgar scores or in admission to neonatal intensive care. These review findings are in contrast to those of the Cochrane review of 2011 [8]. Similarly, we found that although the second stage of labor in the epidural group was longer compared with that in the non-epidural group, the instrumental delivery rate, emergency cesarean section, and neonatal outcomes were similar between the two groups. However, unlike our study, previous studies showed that while a longer second stage of labor may increase vaginal delivery rate, this may be at the expense of increasing maternal and neonatal morbidity [20, 21].

Because of concern of a prolonged second stage of labor and its adverse outcomes, some obstetric nurses ask to reduce or terminate the epidural infusion rate in order to improve the maternal expulsive efforts in the second stage of labor [22]. This practice varies from center to center, but it is reported to occur in $46 \%$ of deliveries (range: $14-85 \%$ ) $[22,23]$. Increased maternal pain or decreased satisfaction with EA may be balanced with the successful benefits of vaginal delivery. However, this is not an ethical solution. Chestnut et al. [24], found that continuous epidural infusion of $0.0625 \%$ bupivacaine had no effect on the duration of the second stage of labor. This finding suggested that there should be a dose-response relationship between the concentration of epidural local anesthetics and the effect of the second stage of labor. These authors' findings are similar to recent findings that minimum local analgesic concentrations of epidural sufentanil or ropivacaine provided satisfactory and safe analgesia for parturients, while they had a low incidence rate of side effects [18, 25].
The potential dose-response mechanism of EA on the success of the second stage of labor needs further study. We used a low concentration of ropivacaine (1\%) with a relative potency of about $0.06 \%$ bupivacaine [26]. We found that the second stage of labor in the epidural group was $34.5 \mathrm{~min}$ (range: 22.8-65.3 $\mathrm{min}$ ) compared with $27.0 \mathrm{~min}$ (range: 18.0-41.3 $\mathrm{min}$ ) in the non-epidural group. Interestingly, we found that the instrumental delivery and emergency cesarean section rates were similar between the epidural and non-epidural groups.

Due to the nature of our research, it has some limitations, which are inherent in retrospective research and may be the cause of some bias. Data, such as maternal satisfaction and dose-response analyses, were missing, which lead to difficultly in further analyzing the underlying reasons and mechanisms of epidural-related maternal or neonatal outcomes. We also did not clarify whether EA is a risk factor, or whether other factors related to analgesia are the causes of adverse outcomes. We plan to add further monitoring in future studies. In addition, the small sample size of this study is a limitation. Therefore, we need to further increase the sample size in the future to further verify our results.

\section{CONCLUSIONS}

EA can prolong the duration of labor, but there is no increase in the normal vaginal delivery rate or incidence of episiotomy, as well as no increase in other adverse effects in mothers or newborns. In summary, EA may be safe for the mother and neonate.

\section{Conflict of interest}

The authors declare no conflict of interest.

\section{Funding}

This research did not receive any specific grant from funding agencies in the public, commercial, or not-for-profit sectors. 


\section{REFERENCES}

1. Hawkins JL. Epidural analgesia for labor and delivery. N Engl J Med. 2010; 362(16): 1503-1510, doi: 10.1056/NEJMct0909254, indexed in Pubmed: 20410515.

2. Jones $L$, Othman $M$, Dowswell $T$, et al. Pain management for women in labour: an overview of systematic reviews. Cochrane Database Syst Rev. 2012(3): CD009234, doi: 10.1002/14651858.CD009234.pub2, indexed in Pubmed: 22419342.

3. Martínez AH, Almagro JJ, García-Suelto MMC, et al. Epidural Analgesia and Neonatal Morbidity: A Retrospective Cohort Study. Int J Environ Res Public Health. 2018; 15(10), doi: 10.3390/ijerph15102092, indexed in Pubmed: 30249991.

4. Herrera-Gómez A, García-Martínez O, Ramos-Torrecillas J, et al. Retrospective study of the association between epidural analgesia during labour and complications for the newborn. Midwifery. 2015; 31(6): 613616, doi: 10.1016/j.midw.2015.02.013, indexed in Pubmed: 25819707.

5. Cheng YW, Shaffer BL, Nicholson JM, et al. Second stage of labor and epidural use: a larger effect than previously suggested. Obstet Gynecol. 2014; 123(3): 527-535, doi: 10.1097/AOG.0000000000000134, indexed in Pubmed: 24499753.

6. Törnell S, Ekéus $C$, Hultin $M$, et al. Low Apgar score, neonatal encephalopathy and epidural analgesia during labour: a Swedish registry-based study. Acta Anaesthesiol Scand. 2015; 59(4): 486-495, doi: 10.1111/aas.12477, indexed in Pubmed: 25683882.

7. Hung TH, Hsieh TT, Liu HP. Differential effects of epidural analgesia on modes of delivery and perinatal outcomes between nulliparous and multiparous women: a retrospective cohort study. PLoS One. 2015; 10(3): e0120907, doi: 10.1371/journal.pone.0120907, indexed in Pubmed: 25807240.

8. Anim-Somuah M, Smyth R, Howell C. Epidural versus non-epidural or no analgesia in labour. Cochrane Database Syst Rev. 2005(4): CD000331, doi: 10.1002/14651858.CD000331.pub2, indexed in Pubmed: 16235275.

9. Laughon SK, Berghella V, Reddy UM, et al. Neonatal and maternal outcomes with prolonged second stage of labor. Obstet Gynecol. 2014; 124(1): 57-67, doi: 10.1097/AOG.0000000000000278, indexed in Pubmed: 24901265.

10. Herrera-Gómez A, De Luna-Bertos E, Ramos-Torrecillas J, et al. Risk Assessments of Epidural Analgesia During Labor and Delivery. Clin Nurs Res. 2018; 27(7): 841-852, doi: 10.1177/1054773817722689, indexed in Pubmed: 28754057.

11. Hasegawa J, Farina A, Turchi G, et al. Effects of epidural analgesia on labor length, instrumental delivery, and neonatal short-term outcome. J Anesth. 2013; 27(1): 43-47, doi: 10.1007/s00540-012-1480-9, indexed in Pubmed: 22965331.

12. Ravelli ACJ, Eskes M, de Groot CJM, et al. Intrapartum epidural analgesia and low Apgar score among singleton infants born at term: A propensity score matched study. Acta Obstet Gynecol Scand. 2020; 99(9): 1155-1162, doi: 10.1111/aogs.13837, indexed in Pubmed: 32142154.

13. Altman $M$, Sandström A, Petersson $G$, et al. Prolonged second stage of labor is associated with low Apgar score. Eur J Epidemiol. 2015; 30(11): 1209-1215, doi: 10.1007/s10654-015-0043-4, indexed in Pubmed: 26008749.
14. Reynolds F. The effects of maternal labour analgesia on the fetus. Best Pract Res Clin Obstet Gynaecol. 2010; 24(3): 289-302, doi: 10.1016/j. bpobgyn.2009.11.003, indexed in Pubmed: 20005180.

15. Wang K, Cao L, Deng $\mathrm{Q}$, et al. The effects of epidural/spinal opioids in labour analgesia on neonatal outcomes: a meta-analysis of randomized controlled trials. Can J Anaesth. 2014; 61(8): 695-709, doi: 10.1007/s12630-014-0185-y, indexed in Pubmed: 25011701.

16. Obstetrics group of anesthesiology society of Chinese medical association. Expert consensus on labor analgesia. J Clin Anesthesiol. 2016; 32: 816-818.

17. Wang $\mathrm{Q}$, Zheng SX, Ni YF, et al. The effect of labor epidural analgesia on maternal-fetal outcomes: a retrospective cohort study. Arch Gynecol Obstet. 2018; 298(1): 89-96, doi: 10.1007/s00404-018-4777-6, indexed in Pubmed: 29777348.

18. Sun J, Yan X, Yuan A, et al. Effect of epidural analgesia in trial of labor after cesarean on maternal and neonatal outcomes in China: a multicenter prospective cohort study. BMC Pregnancy Childbirth. 2019; 19(1): 498, doi: 10.1186/s12884-019-2648-1, indexed in Pubmed: 31842795.

19. Anim-Somuah M, Smyth RMd, Cyna AM, et al. Epidural versus non-epidural or no analgesia in labour. Cochrane Database Syst Rev. 2011; 5(12): CD000331, doi: 10.1002/14651858.CD000331.pub3, indexed in Pubmed: 22161362.

20. Spong CY, Berghella V, Wenstrom KD, et al. Preventing the first cesarean delivery: summary of a joint Eunice Kennedy Shriver National Institute of Child Health and Human Development, Society for Maternal-Fetal Medicine, and American College of Obstetricians and Gynecologists Workshop. Obstet Gynecol. 2012; 120(5): 1181-1193, doi: 10.1097/aog.0b013e3182704880, indexed in Pubmed: 23090537.

21. Rouse D, Weiner S, Bloom S, et al. Second-stage labor duration in nulliparous women: relationship to maternal and perinatal outcomes. American Journal of Obstetrics and Gynecology. 2009; 201(4): 357.e1-357.e7 doi: 10.1016/j.ajog.2009.08.003.

22. Roberts $\mathrm{CL}$, Raynes-Greenow $\mathrm{CH}$, Upton $\mathrm{A}$, et al. Management of labour among women with epidural analgesia. Aust N Z J Obstet Gynaecol. 2003; 43(1): 78-81, doi: 10.1046/j.0004-8666.2003.00018.x, indexed in Pubmed: 12755355 .

23. Shen X, Li Y, Xu S, et al. Epidural Analgesia During the Second Stage of Labor: A Randomized Controlled Trial. Obstet Gynecol. 2017; 130(5): 1097-1103, doi: 10.1097/AOG.0000000000002306, indexed in Pubmed: 29016499.

24. Chestnut DH, Laszewski LJ, Pollack KL, et al. Continuous epidural infusion of $0.0625 \%$ bupivacaine- $0.0002 \%$ fentanyl during the second stage of labor. Anesthesiology. 1990; 72(4): 613-618, doi: 10.1097/00000542199004000-00006, indexed in Pubmed: 2321776.

25. Zeng $H$, Guo F, Lin B, et al. The effects of epidural analgesia using low-concentration local anesthetic during the entire labor on maternal and neonatal outcomes: a prospective group study. Arch Gynecol Obstet. 2020; 301(5): 1153-1158, doi: 10.1007/s00404-020-05511-8, indexed in Pubmed: 32219521.

26. Capogna G, Celleno D, Fusco P, et al. Relative potencies of bupivacaine and ropivacaine for analgesia in labour. Br J Anaesth. 1999; 82(3): 371-373, doi: 10.1093/bja/82.3.371, indexed in Pubmed: 10434818. 